\title{
Autoimmune polyendocrinopathy type 2
}

INSERM

\section{Source}

INSERM. (1999). Orphanet: an online rare disease and orphan drug data base.

Autoimmune polyendocrinopathy type 2. ORPHA:3143

Autoimmune polyendocrinopathy type 2 is a rare, endocrine disease characterized by autoimmune Addison disease associated with autoimmune thyroid disease or type I diabetes mellitus, or both, and without chronic candidiasis. Additional endocrine (hypogonadism, hypoparathyroidism) and non-endocrine diseases (vitiligo, autoimmune hepatitis, autoimmune gastritis, pernicious anemia, and myasthenia gravies) may be present. 